\title{
Local complex dimensions of a fractal string
}

\author{
Jacques Lévy Véhel, Franklin Mendivil ${ }^{\dagger}$
}

August 13, 2011

\begin{abstract}
We investigate in this work a local version of the theory of fractal strings and associated geometric zeta functions. Such a generalization allows to describe the asymptotic behaviour of a "fractal" set in the neighborhood of any of its points. We give basic properties and several examples illustrating the possible range of situations concerning in particular the evolution of the local complex dimensions along the set and the relation between local and global zeta functions.
\end{abstract}

\section{Background and Motivations}

The theory of Fractal Strings has been developed over the past years by M. Lapidus and co-workers in a series of papers, including [7, 8, 9, 12, 13, 14, 5]. See also the book [10] and the more recent works $[11,15,16]$.

A fractal string $\mathcal{L}$ is simply a bounded open subset $\Omega$ of $\mathbb{R} . \quad \Omega$ may be written as a disjoint union of intervals $I_{j}=\left(a_{j}, b_{j}\right)$, i.e. $\Omega=\bigcup_{j=1}^{\infty} I_{j}$. For our purpose, no distinction needs to be made between the open set and the sequence of lengths of the intervals that define it. By abuse of notation, we will thus speak of the fractal string as $\mathcal{L}=\left\{\ell_{j}\right\}_{j=1}^{\infty}$, where $\ell_{j}$ are the lengths of the $I_{j}$. Thus, a possible definition of a fractal string is:

Definition 1 A fractal string $\mathcal{L}$ is an at-most countable, non-increasing sequence of lengths whose sum is finite.

To a fractal string is associated a complex function, the (geometric) zeta function of $\mathcal{L}$ :

Definition 2 The geometric zeta function of a fractal string $\mathcal{L}$ is

$$
\zeta_{\mathcal{L}}(s)=\sum_{j=1}^{\infty} \ell_{j}^{s}=\sum_{n=1}^{\infty} m_{n} l_{n}^{s}
$$

for values $s \in \mathbb{C}$ such that the series converges.

\footnotetext{
*Inria, Regularity team, Parc Orsay Université, 4 rue J. Monod, 91893 Orsay Cedex France, jacques.levy-vehel@inria.fr

${ }^{\dagger}$ Department of Mathematics and Statistics, Acadia University, 12 University Avenue, Wolfville, NS Canada B4P 2R6, franklin.mendivil@acadiau.ca
} 
The interest of $\zeta_{\mathcal{L}}$ is that it provides rich information about the fractal structure of the boundary $\partial \Omega$ of $\Omega$ (or of $\mathcal{L}$ for our purpose), as is developed in the papers mentioned above. The most basic fact is that the critical exponent for $\zeta_{\mathcal{L}}$ is related to the upper Minkowski dimension (or upper box dimension) of $\partial \Omega^{1}$. Recall the following definition of the upper Minkowski dimension:

The one-sided volume of the tubular neighborhood of radius $\varepsilon$ of $\partial \Omega$ is

$$
V(\varepsilon)=\operatorname{vol}_{1}\{x \in \Omega \mid d(x, \partial \Omega)<\varepsilon\} .
$$

The Minkowski dimension of $\partial \Omega$ is

$$
D=D_{\mathcal{L}}:=\inf \left\{\alpha \geq 0 \mid V(\varepsilon)=O\left(\varepsilon^{1-\alpha}\right) \text { as } \varepsilon \rightarrow 0^{+}\right\} .
$$

It is a remarkable fact that only the lengths of $\Omega$ (that is, of the associated fractal string $\mathcal{L}$ ) affect its value. It is thus relevant to speak of the Minkowski dimension of $\partial \mathcal{L}$. By abuse of notation, we will often speak of the dimension of $\mathcal{L}$ instead of $\partial \mathcal{L}$.

If $D$ is the Minkowski dimension and $\lim _{\varepsilon \rightarrow 0^{+}} V(\varepsilon) \varepsilon^{D-1}$ exists $\mathcal{L}$ is said to be Minkowski measurable, in which case

$$
D=\inf \left\{\alpha \geq 0 \mid \lim _{\varepsilon \rightarrow 0^{+}} V(\varepsilon) \varepsilon^{\alpha-1}<\infty\right\} .
$$

The Minkowski content of $\mathcal{L}$ is then defined as $\mathcal{M}(D, \mathcal{L})=\lim _{\varepsilon \rightarrow 0^{+}} V(\varepsilon) \varepsilon^{D-1}$.

The following theorem describes the relation between the Minkowski dimension of a fractal string $\mathcal{L}$ and the sum of its lengths to the exponent $\sigma \in \mathbb{R}$.

Theorem 1 [10]

$$
D=\inf \left\{\sigma \in \mathbb{R} \mid \sum_{j=1}^{\infty} \ell_{j}^{\sigma}<\infty\right\}
$$

This sum is monotonically decreasing (as a function of $\sigma$ ), so there is a unique such $D \in \mathbb{R}$. We see that $D$ is the abscissa of convergence of $\zeta_{\mathcal{L}}(s)$, where $s \in \mathbb{C}$.

A crucial assumption in all that follows is that the zeta function $\zeta_{\mathcal{L}}$ has a meromorphic extension to an open region of the complex plane which extends strictly to the left of line $\mathcal{R}(z)=D$. By an abuse of notation, we use the same notation for the zeta function and its meromorphic extension.

Thus, the number $D$ is a real pole of $\zeta_{\mathcal{L}}$ and it is the Minkowski dimension of $\mathcal{L}$. This suggests to extend the notion of dimension to complex numbers by considering the poles of the meromorphic extension of $\zeta_{\mathcal{L}}$ :

Definition 3 The set of complex dimensions of a fractal string $\mathcal{L}$ contained in some region $R \subseteq \mathbb{C}$ is

$$
\mathcal{D}(R)=\left\{\omega \in R \mid \zeta_{\mathcal{L}} \text { has a pole at } \omega\right\} .
$$

\footnotetext{
${ }^{1}$ Since we will only deal with the upper Minkowski dimension in this work, we will often leave out the word "upper" in the name.
} 
(See [10] for a more general definition.) The relevance of considering complex dimensions is illustrated in particular by the following theorem :

Theorem 2 [10] If a fractal string satisfies certain mild conditions, the following are equivalent:

1. $\mathcal{L}$ has only one complex dimension with real part equal to $D_{\mathcal{L}}$.

2. $\partial \mathcal{L}$ is Minkowski measurable.

See [10] for more details. More refined results exist, that allow for instance to characterize the asymptotic behavior of $V(\varepsilon)$ (as $\varepsilon$ tends to 0 ) in terms of the residues of $\zeta_{\mathcal{L}}$.

Example: A simple example will best illuminate and motivate these definitions. The example we choose is the standard middle- $1 / 3$ Cantor subset of $[0,1]$. See reference [10] pages 13-16 for a more complete discussion of this example. The lengths for this fractal string are

$$
1 / 3,1 / 9,1 / 9,1 / 27,1 / 27,1 / 27,1 / 27,1 / 81, \ldots, 1 / 81,1 / 243, \ldots
$$

with $2^{n-1}$ copies of $3^{-n}$ for $n \geq 1$. It is easy to see that

$$
\zeta_{\mathcal{L}}(s)=\frac{1 / 3}{1-2 \cdot 3^{-s}}
$$

so that $\zeta_{\mathcal{L}}$ has a meromorphic extension to all of $\mathbb{C}$. Thus, the poles of $\zeta_{\mathcal{L}}(s)$ are the points

$$
\frac{\ln (2)}{\ln (3)}+\frac{2 \pi i k}{\ln (3)}, \quad k=0, \pm 1, \pm 2, \pm 3, \ldots
$$

In this particular case, we have

$V(\epsilon)=2 \epsilon \cdot\left(2^{n}-1\right)+(2 / 3)^{n}-2 \epsilon=(2 \epsilon)^{1-D}\left((1 / 2)^{\left\{-\log _{3}(2 \epsilon)\right\}}+(3 / 2)^{\left\{-\log _{3}(2 \epsilon)\right\}}\right)-2 \epsilon$,

where $n=\left[-\log _{3}(2 \epsilon)\right]$ and $x=[x]+\{x\}$ is the decomposition of a positive real number into its integer and fractional parts and $D=\ln (2) / \ln (3)$ is the dimension of the Cantor set. This means that

$$
V(\epsilon)=\epsilon^{1-D} G(\epsilon)-2 \epsilon
$$

where $G$ is a non-constant multiplicatively periodic function of multiplicative period $2 \pi / \ln (3)$ (see [10], page 16). Notice the connection between the interpole spacing of $\zeta_{\mathcal{L}}(s)$ and the oscillations of $V(\epsilon)$ (that is, there is the quantity $2 \pi / \ln (3)$ in formulas (1) and (2)).

The theory of fractal strings has been generalized in various directions: a random setting is considered in [5]; higher-dimensional extensions are proposed in $[13,16]$; and multifractal zeta functions are investigated in $[11,15,16]$.

The aim of this article is to study a localized version of the geometric zeta function tailored to measure the dimensional properties of a fractal set in the 
neighbourhood of any of its points. By definition, the local behaviour of a self-similar fractal $E$ is the same everywhere (for example, $\operatorname{dim}_{H}\left(E \cap B_{\epsilon}(x)\right)=$ $\operatorname{dim}_{H}(E)$, as long as this intersection is non-empty, where $B_{\epsilon}(x)$ is the open ball of radius $\epsilon$ centered at $x$ ). Thus if the local dimension differs at different points we know that the set cannot be self-similar and thus may exhibit different scaling behaviour at different points. It is natural to wonder whether localizing the formalism of fractal strings and geometric zeta functions may help in characterizing the fine pointwise behaviour of non-self-similar fractal sets, as for instance local Minkowski measurability. This is what we develop in the following. Note that this work is meant as a preliminary investigation: we content ourselves with defining local fractal strings in a meaningful way (section 2), proving basic properties (section 3), and giving a variety of examples that illustrate the range of possible behaviours (section 4).

\section{Local fractal strings}

In all the sequel, $C \subset[0,1]$ will denote a compact set, so that $[0,1] \backslash C=\bigcup_{i} l_{i}$ is a union of open intervals. By abuse of language, we will occasionally refer to $C$ as a string, instead of the bounded components of its complement.

Definition 4 Let $x \in C$ and $\epsilon>0$ be given. We define

$$
\zeta_{\epsilon}(x, s)=\sum_{n: l_{n} \cap B_{\epsilon}(x) \neq \emptyset}\left|l_{n}\right|^{s}
$$

(where $\left|l_{n}\right|$ is the length of the interval $l_{n}$ ) as the $\epsilon$-localization of the zeta function around the point $x \in C$. We denote by $D_{\epsilon}(x)=\inf \left\{s>0: \zeta_{\epsilon}(x, s)<\right.$ $\infty\}$, the exponent of convergence of $\zeta_{\epsilon}(x, s)$.

We assume that $\zeta_{\epsilon}(x, s)$ has a meromorphic extension to a neighborhood of $\left\{z: \operatorname{Re}(z) \geq D_{\epsilon}(x)\right\}$ for all sufficiently small $\epsilon$. We continue the tradition of abusing notation by denoting both the original zeta function and its meromorphic extension by $\zeta_{\epsilon}(x, s)$.

Definition 5 The $\epsilon$-localization of the complex dimensions of the fractal string $\left\{l_{n}\right\}$ is the set of poles of this meromorphic extension of $\zeta_{\epsilon}(x, s)$ on the critical line. We denote this set of poles by $\mathcal{D}_{\epsilon}(x)$.

We will be interested in the set $\mathcal{D}(x)=\lim _{\epsilon} \mathcal{D}_{\epsilon}(x)$ and when this limit exists. $\mathcal{D}_{\epsilon}(x)$ is a collection of points on the line in $\mathbb{C}$ with real part $D_{\epsilon}(x)$, we need to specify in which sense we take this limit.

Definition 6 Let $K_{n}$ be closed subsets of $\mathbb{C}$ with $K_{n}$ being on the line with real part $s_{n}$ and similarly $K$ with real part $s$. We say that $K_{n} \rightarrow K$ if $s_{n} \rightarrow s$ and for every compact set $A$ we have $A \cap K_{n} \rightarrow A \cap K$ in the Hausdorff metric.

In particular, notice that this means that any bounded subset of poles converges uniformly. 
Definition 7 The local complex dimensions at the point $x \in C$ for the fractal string $\left\{l_{n}\right\}$ is the set $\mathcal{D}(x)$, when this limit exists.

Remark that we do not define

$$
\zeta(x, s)=\lim _{\epsilon \rightarrow 0} \zeta_{\epsilon}(x, s)=\inf _{\epsilon>0} \zeta_{\epsilon}(x, s)
$$

since for all but countably many points in $C$ (the endpoints of the $l_{n}$ ) we have that

$$
\lim _{\epsilon \rightarrow 0} \zeta_{\epsilon}(x, s)=0
$$

uniformly in $s$ on compact subsets. This is why we first define $\mathcal{D}_{\epsilon}(x)$ and then take the limit of the complex dimensions. This is similar to the usual definition of the local dimension

$$
\operatorname{dim}^{l o c}(C, x)=\lim _{\epsilon \rightarrow 0} \operatorname{dim}\left(C \cap B_{\epsilon}(x)\right)
$$

and not

$$
\operatorname{dim}^{l o c}(C, x)=\operatorname{dim}\left(\lim _{\epsilon \rightarrow 0} C \cap B_{\epsilon}(x)\right)=0 .
$$

In a similar way

$$
D(x)=\inf _{\epsilon>0} \inf \left\{s>0: \zeta_{\epsilon}(x, s)<\infty\right\}
$$

and not

$$
\inf \left\{s>0: \inf _{\epsilon>0} \zeta_{\epsilon}(x, s)<\infty\right\}=0 .
$$

\section{$3 \quad$ Basic properties}

We first remark that, for a fractal string $\left\{l_{n}\right\}$ and a point $x$, it is not usually the case that $\mathcal{D}(x) \subset \mathcal{D}$, that is the set of local complex dimensions is not, in general, a subset of the set of global complex dimensions (example 3 in the next section is one illustration of this).

Proposition 1 The quantity

$$
D(x)=\lim _{\epsilon \rightarrow 0} D_{\epsilon}(x)
$$

always exists and is the local Minkowski dimension (or local upper box dimension) of $C$ at $x$.

Proof: The sets $\left\{i: l_{i} \cap B_{\epsilon}(x)\right\}$ are increasing as functions of $\epsilon$, thus

$$
D(x)=\inf _{\epsilon>0} D_{\epsilon}(x)
$$

The fact that $D(x)$ is the local Minkowski dimension is by definition. 
Proposition $2 D(x)$ is an upper semi-continuous function of $x \in C$.

Proof: $\quad$ Take $x \in C$. Then for any $\epsilon>0$ and $z$ so that $|z-x|<\epsilon$ we have

$$
D(z) \leq D_{\epsilon-|x-z|}(z) \leq D_{\epsilon}(x)
$$

and so

$$
\sup _{|z-x|<\epsilon} D(z) \leq \sup _{|z-x|<\epsilon} D_{\epsilon-|x-z|}(z) \leq D_{\epsilon}(x)
$$

which means that

$$
\limsup _{z \rightarrow x} D(z)=\inf _{\epsilon>0} \sup _{|z-x|<\epsilon} D(z) \leq \inf _{\epsilon>0} \sup _{|z-x|<\epsilon} D_{\epsilon-|x-z|}(z) \leq \inf _{\epsilon>0} D_{\epsilon}(x)=D(x) .
$$

Theorem 3 in section 4 will provide a converse to proposition 2 .

Proposition 3 There exists at least one point $x \in C$ with $D(x)$ equal to the global Minkowski dimension of the string.

\section{Proof:}

Let $D$ denote the (global) Minkowski dimension. Then clearly we have $D(x) \leq D$ for all $x$. Suppose $D(x)<D$ for all $x$. For any $x_{i}$, there is some $\epsilon_{i}>0$ so that $D_{\epsilon_{i}}\left(x_{i}\right)<\left(D+D\left(x_{i}\right)\right) / 2$. As $C$ is compact, we can cover it with finitely many $B_{\epsilon_{i}}\left(x_{i}\right)$. Let $j$ be such that $D_{\epsilon_{j}}\left(x_{j}\right)$ is the maximum amongst the $D_{\epsilon_{i}}\left(x_{i}\right)$. Then as the Minkowski dimension is finitely stable we have that

$$
D \leq D_{\epsilon_{j}}\left(x_{j}\right)<\left(D+D\left(x_{j}\right)\right) / 2<D,
$$

a contradiction. $\square$

Our final result in this section gives a criterion for the existence of the local complex dimensions:

Proposition 4 Let $C$ be a string and suppose that $x \in C$ is such that $D(x)$ has a strict local maximum at $x$. Then the local complex dimensions $\mathcal{D}(x)$ of $C$ at $x$ exist.

Proof: $\quad$ Since $D(x)>D(y)$ for any $y$ sufficiently close to $x$, this implies that for all small $0<\delta<\epsilon$, we have $\mathcal{D}_{\epsilon}(x)=\mathcal{D}_{\delta}(x)$. This entails convergence as $\epsilon \rightarrow 0$, as this set is constant.

It is interesting to compare this result with example 8, where the local complex dimensions do not exist at a point because of a lack of convergence. In that example the point in question is a strict local minimum of $D(x)$. 


\section{Examples of local fractal strings}

Our first example is just to given an example of the simplest type of behaviour that can occur when we localize a fractal string.

\section{Example 1}

Take the intervals $l_{n}=(1 /(n+1), 1 / n)$ for $n \in \mathbb{N}$. For $x=1 / n$, we have

$$
\zeta_{\epsilon}(x, s)=[n(n-1)]^{-s}+[(n+1) n]^{-s}
$$

for all $\epsilon<\frac{1}{n(n+1)}$, so this zeta function has no poles and $D(x)=0$, as expected.

For $x=0$, we have that

$$
\zeta_{\epsilon}(0, s)=\sum_{n>1 / \epsilon}[n(n-1)]^{-s} .
$$

Clearly $D=1 / 2$ in this case. Furthermore, the asymptotic decay rate of $\zeta_{\epsilon}(0, s)$ does not depend on $\epsilon>0$, so $\mathcal{D}_{\epsilon}(x)$ is constant in $\epsilon$. For a detailed discussion of this fractal string, see [10] Section 6.5.1.

\section{Example 2 Self-similar local fractal string}

Take a fractal string generated by an IFS $w_{i}(x)=r_{i} x+a_{i}$ on $[0,1]$ with gaps of length $g_{i}$ (that is, $[0,1] \backslash \cup_{i} w_{i}([0,1])$ is a union of open intervals with lengths $g_{i}$ ). The usual (non-local) zeta function $\zeta_{\mathcal{L}}(s)$ of this string satisfies the functional equation

$$
\zeta_{\mathcal{L}}(s)=\sum_{i} g_{i}^{s}+\sum_{i} r_{i}^{s} \zeta_{\mathcal{L}}(s)
$$

from which we obtain

$$
\zeta_{\mathcal{L}}(s)=\frac{\sum_{i} g_{i}^{s}}{1-\sum_{i} r_{i}^{s}} .
$$

Now, for the local fractal string, let $x \in C$, the Cantor set generated by this IFS, and $\epsilon>0$ be given. Then there is an $n$ and some sequence $i_{1}, i_{2}, \ldots, i_{n}$ with

$$
w_{i_{1}} \circ w_{i_{2}} \circ \cdots w_{i_{n}}(C) \subset B_{\epsilon}(x)
$$

and thus

$$
\zeta_{\epsilon}(x, s)=\left(r_{i_{1}} r_{i_{2}} \cdots r_{i_{n}}\right)^{s} \zeta_{\mathcal{L}}(s)+\sum \text { other terms. }
$$

Thus, all the poles of $\zeta_{\mathcal{L}}(s)$ are also poles of $\zeta_{\epsilon}(x, s)$. We already observed that all the poles of $\zeta_{\epsilon}(x, s)$ are also poles of $\zeta_{\mathcal{L}}(s)$. Thus, $\mathcal{D}_{\epsilon}(x)$ is simply the set of poles of the non-local zeta function $\zeta_{\mathcal{L}}(s)$ and is thus constant with respect to $\epsilon$.

So, as we expect, the behaviour of the local fractal string for a self-similar fractal is constant everywhere and is just measuring the global behaviour of the string. 
Example 3 Continuously varying $D(x)$.

Now we construct $C \subset[0,1]$, a Cantor set, where the local Minkowski dimension (in fact, Hausdorff dimension) continuously varies from 0 to $\log (2) / \log (3)$ (the reason for the $\log (2) / \log (3)$ factor will become a little more clear when we revisit this example in example 5).

To do this, we first discuss a general method of constructing compact, totally disconnected and perfect subsets of $[0,1]$ (studied also in [2]).

Let $\left(a_{n}\right)$ be a positive summable sequence, assuming without loss of generality that $\sum_{n} a_{n}=1$. We construct a Cantor set $C_{a}$ with gap lengths $a_{n}$ by first removing an interval of length $a_{1}$ from the interior of $[0,1]$, leaving two closed intervals $I_{1}^{1}, I_{2}^{1}$. Having constructed the $k$ th step, we have $2^{k}$ closed intervals $I_{i}^{k}$ and we remove from the interior of $I_{i}^{k}$ an open interval of length $a_{2^{k}+i-1}$ (see the figure below). Because of the way the intervals are removed, there is only one location possible for each "gap" (for example, the gap of length $a_{1}$ begins at the point

$$
\sum_{k=1}^{\infty} \sum_{i=0}^{2^{k-1}-1} a_{2^{k}+i}
$$

the sum over the "left half" of the binary tree).

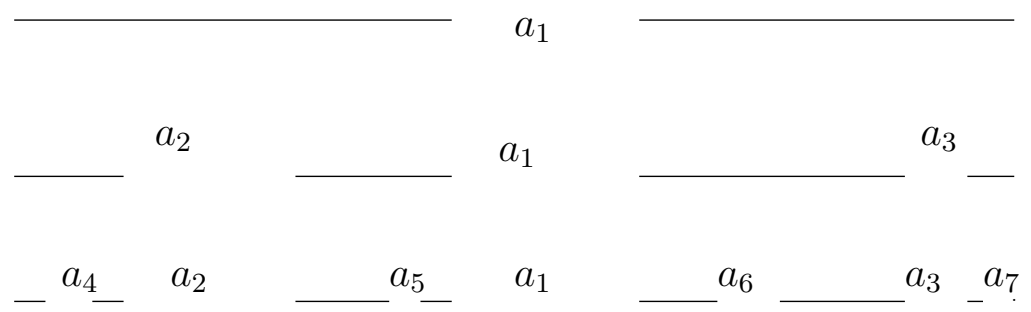

Notice that the order of the sequence $\left(a_{n}\right)$ can make a big difference in the resulting Cantor set. Further, the sequence determines the Cantor set but there are many sequences which result in the same Cantor set. We mention that there are relations between the decay rate of $\left(a_{n}\right)$ and the dimension of the associated Cantor set (see $[1,2])$.

We will specify a summable sequence $a_{n}$ in a particular order and this will generate the desired Cantor set.

Let let $\Sigma^{0}=\emptyset$ and $\Sigma^{n}=\{0,1\}^{n}$, for $n \geq 1$. We also let $w(\emptyset)=1$ and $w(\sigma)=\sum_{i} \sigma_{i} 2^{-i}+2^{-n-1}$ for $\sigma \in \Sigma^{n}$. Further, for each $\sigma \in \Sigma^{n}$, we define

$$
l(\sigma)=2^{-(n \log (3)) /(w(\sigma) \log (2))}=3^{-n / w(\sigma)} .
$$


Finally, we let

$$
N(\sigma)=2^{n}+\sum_{i=1}^{n} \sigma_{i} 2^{i}
$$

(so $N(\emptyset)=1, N(0)=2, N(1)=3, N(00)=4, N(01)=5$ etc) and notice that $N: \cup_{n} \Sigma^{n} \rightarrow \mathbb{N}$ is a bijection.

Using this notation, we define $a_{N(\sigma)}=l(\sigma)$.

By our construction, each $x \in C$ is identified with a unique (infinite) binary sequence $\sigma(x)=\sigma_{1}(x) \sigma_{2}(x) \sigma_{3}(x) \ldots$. In fact, if we place the product topology on $\Sigma^{\infty}=\{0,1\}^{\infty}$ we get that this identification is a homeomorphism between $C$ and $\Sigma^{\infty}$. We extend the function $w$ (given above as $\left.w: \Sigma^{n} \rightarrow[0,1]\right)$ to $w: \Sigma^{\infty} \rightarrow[0,1]$ by

$$
w(\sigma)=\sum_{n} \sigma_{n} 2^{n}
$$

(so this is just binary representation of numbers in $[0,1]$ ).

We will show that $D(x)=\log (3) /(\log (2) w(\sigma(x)))$ for any $x \in C$.

Roughly speaking, we define $l(\sigma)=2^{-n \log (3) /(\log (2) w(\sigma))}$ because we want the decay rate near some $x \in C$ to be approximately $n^{-\log (3) /(\log (2) w(\sigma(x)))}$. That is, if $\sigma(x)=\sigma_{1} \sigma_{2} \sigma_{3} \ldots \sigma_{n} \ldots$, then we see that for large $n, w(\sigma(x)) \approx$ $w\left(\sigma_{1} \sigma_{2} \ldots \sigma_{n}\right)$. Let $\sigma^{n}=\sigma_{1} \sigma_{2} \ldots \sigma_{n}$ be the truncation of $\sigma$ to $n$ places. Then

$$
\frac{l\left(\sigma^{n+1}\right)}{l\left(\sigma^{n}\right)}=\frac{2^{-(n+1) \log (3) /\left(\log (2) w\left(\sigma^{n+1}\right)\right)}}{2^{-n \log (3) /\left(\log (2) w\left(\sigma^{n}\right)\right)}} \approx 2^{-\log (3) /(\log (2) w(\sigma))}
$$

so that this definition gives the correct local decay rate.

A little more formally, for $x \in C$ and $\epsilon>0$, define

$$
w_{\min }=\inf \left\{w(\sigma(z)): z \in B_{\epsilon}(x)\right\} \quad \text { and } \quad w_{\max }=\sup \left\{w(\sigma(z)): z \in B_{\epsilon}(x)\right\} .
$$

and $\Omega=\sigma^{-1}\left(B_{\epsilon}(x)\right) \subset \Sigma^{\infty}$. Then for any $\sigma \in \Omega$ we have

$$
2^{-n \log (3) /\left(\log (2) w_{\max }\right)} \leq l\left(\sigma^{n}\right) \leq 2^{-n \log (3) /\left(\log (2) w_{\min }\right)} .
$$

This means that the decay rate of the sequence associated with local Cantor set $C \cap B_{\epsilon}(x)$ is bounded between $n^{-\log (3) /\left(\log (2) w_{\max }\right)}$ and $n^{-\log (3) /\left(\log (2) w_{\min }\right)}$. By the results in [2] (in particular Proposition 3), this means that

$\log (3) /\left(\log (2) w_{\max }\right) \leq \operatorname{dim}_{H}\left(C \cap B_{\epsilon}(x)\right) \leq \operatorname{dim}_{B}\left(C \cap B_{\epsilon}(x)\right)=D_{\epsilon}(x) \leq \log (3) /\left(\log (2) w_{\min }\right)$.

Since both $w$ and $\sigma$ are continuous functions, as $\epsilon \rightarrow 0$, we have $w_{\text {min }}, w_{\max } \rightarrow$ $w(\sigma(x))$ and thus $D(x)=\log (3) /(\log (2) w(\sigma(x)))$, as desired. In fact, in this case the "local Hausdorff" dimension is also $D(x)$, as our estimate bounds the Hausdorff dimension of $B_{\epsilon}(x) \cap C$. 2:

Example 3 may be somewhat generalized to give a converse to proposition 
Theorem 3 Let $f:[0,1] \rightarrow[0,1)$ be an upper semi-continuous function. Let $w: \Sigma \rightarrow[0,1]$ give the binary representation (as above). Then there exists a Cantor set $C$ so that for each $x \in C$, the local Minkowski dimension $D(x)$ at $x$ is $f(w(\sigma))$, where $\sigma \in \Sigma$ is the address of $x$.

\section{Proof:}

The construction is very much the same as in example 3 . We only make a very slight modification to the lengths and then the estimates are a little different. For each $\sigma \in \Sigma^{n}$, let the interval

$$
I_{\sigma}=\left[\sum_{i=1}^{n} \sigma_{i} 2^{-i}, 2^{-n}+\sum_{i=1}^{n} \sigma_{i} 2^{-i}\right] \subset[0,1],
$$

so that $I_{\sigma}$ is a dyadic interval of length $2^{-n}$. Further, let $f_{\sigma}=\sup \{f(x): x \in$ $\left.I_{\sigma}\right\}$. Then if $\sigma \in \Sigma$ with $w(\sigma)=x$, then because $f$ is u.s.c. we know that $f(x)=\lim _{n} f_{\sigma^{n}}$ (recall that $\sigma^{n}$ is the truncation of $\sigma$ to the first $n$ places). It is also useful to note that

$$
f_{\sigma}=\max \left\{f_{\alpha}: \alpha \in \Sigma^{n+k}, \alpha^{n}=\sigma\right\}
$$

for any $k \in \mathbb{N}$.

Referring to the construction in example 3 , we now define $l(\sigma)=2^{-n / f_{\sigma}}$ and $a_{N(\sigma)}=l(\sigma)$ and let $C$ be the resulting Cantor set.

We now make use of the following result from [18] to estimate the Minkowski (upper box) dimension $D$ :

$$
D=\limsup \frac{\ln (n)}{-\ln \left(a_{n}\right)}=\inf \left\{s>0: \sum_{n} a_{n}^{s}<\infty\right\} .
$$

Take $x \in C$ and $\sigma \in \Sigma^{n}$ be such that $x \in I_{\sigma}$. Then for all $k \in \mathbb{N}$, there is some $\alpha_{k} \in \Sigma^{n+k}$ with $f_{\alpha_{k}}=f_{\sigma}$. We have $N\left(\alpha_{k}\right)=\eta 2^{n+k}$ for some $1 \leq \eta<2$ and $a_{N\left(\alpha_{k}\right)}=2^{-(n+k) / f_{\sigma}}$. Thus for all $k$ we have that

$$
\frac{\ln \left(N\left(\alpha_{k}\right)\right)}{-\ln \left(a_{N\left(\alpha_{k}\right)}\right)}=\frac{\ln (\eta) f_{\sigma}}{(n+k) \ln (2)}+f_{\sigma}>f_{\sigma}
$$

and thus for the lengths in $I_{\sigma}$ we have that $\lim \sup \frac{\ln (n)}{-\ln \left(a_{n}\right)} \geq f_{\sigma}$. From the same argument as in the example we have $f_{\sigma}$ as an upper bound and thus $D_{I_{\sigma}}(x)=f_{\sigma}$. Letting $n \rightarrow \infty$ we converge to $x$ and to $f(w(\sigma))$, as announced.

\section{Example 4}

It is possible to modify the construction of the previous example so that all the lengths are powers of any given $0<r<1$. To do this, simply define

$$
\hat{l}(\sigma)=r^{k(\sigma)}
$$


with

$$
k(\sigma)=\left\lceil-\left(\frac{n \log (2) \log (3)}{w(\sigma(x)) \log (2) \log (r)}\right)\right\rceil=\left\lceil-\left(\frac{n \log (3)}{w(\sigma(x)) \log (r)}\right)\right\rceil .
$$

This will give the same values for $D(x)$ for the local zeta functions; the complex dimensions need not be the same.

Example 5 Rearranging the middle third Cantor string

Now we construct an example which has continuously varying $D(x)$ (as example 3 ) but in which the lengths are a subset of the lengths of the standard middle third Cantor string.

Take the string from example 3 but modify them as in example 4 by taking $r=1 / 3$. This means that all the lengths are negative powers of 3 . Call the lengths of the resulting string $l_{n}$ and the entire string $\mathcal{L}$. We show that it is possible to rearrange the intervals in the middle third Cantor string and obtain the string $\mathcal{L}$ plus some "left-over" lengths. We arrange these "extra" lengths as in example 1, thus giving this part of the string trivial local dimension except at one point. Since we are primarily interested in the remainder of the string, we will ignore these "extra" lengths.

The middle third Cantor string has $2^{k}$ intervals of length $3^{-k-1}$. Let $N_{k}$ be the number of lengths in $\mathcal{L}$ equal to $3^{-k}$. We estimate that $N_{k} \leq 2^{k}$. Thus there is a re-scaling of $\mathcal{L}$, call it $\widehat{\mathcal{L}}$ whose lengths are a subset of the lengths of the middle third Cantor string, as desired.

To estimate $N_{k}$, we define

$$
S_{k}^{n}=\left\{x \in[0,1]: \frac{n}{k} \leq x<\frac{n}{k-1}\right\},
$$

noting that $S_{k}^{n}$ is roughly the set of $\sigma \in \Sigma^{n}$ so that $k(\sigma)=k(k(\sigma)$ as defined in (3)). The set $S_{k}^{n}$ represents roughly $2^{n}\left|S_{k}^{n}\right|$ terms of size $3^{-k}$, so we estimate that

$$
N_{k}=\sum_{n=1}^{k-1} 2^{n} \frac{n}{k(k-1)}=\left(\frac{k-2}{k(k-1)}\right) 2^{k}+\frac{2}{k(k-1)}
$$

so that $N_{k} \leq 2^{k}$ as desired.

Example 6 A string with different local and global oscillations

To begin this example, we first briefly discuss a self-similar string with a single gap (see Section 2.2.1 in [10]). For our purposes, we take the single gap $\left(0,3^{-n}\right)$ and $2^{n}$ IFS maps of the form $w_{i}(x)=3^{-n} x+i / 3^{n}$ for $i=1,2, \ldots, 2^{n}$. The zeta function of this self-similar string is

$$
\zeta_{n}(s)=\frac{3^{-n s}}{1-2^{n} 3^{-n s}}
$$


with complex poles at the points

$$
\frac{\ln (2)}{\ln (3)}+\frac{2 \pi i k}{n \ln (3)}, \quad k \in \mathbb{Z} .
$$

We will call this fractal string the $3^{-n}$ string. For each $k \in \mathbb{N}$, it contains $2^{n k}$ copies of the length $3^{-n(k+1)}$. The length of this string is $3^{-n} /\left(1-2^{n} 3^{-n}\right)=$ $1 /\left(3^{n}-2^{n}\right)$.

Let $\mathcal{L}$ be a disjoint union of a $3^{-n}$ string for each $n \in \mathbb{N}$, arranged in order on $\mathbb{R}$ and going from left to right (the total length of $\mathcal{L}$ is less than $3 / 2$ ).

Consider the length $3^{-j}$ for some $j$. We see that this length occurs $2^{j-1}$ times in the standard middle $1 / 3$ Cantor string. If this length occurs in some $3^{-n_{1}}$ and $3^{-n_{2}}$ string with $2^{k_{1}}$ copies and $2^{k_{2}}$ copies, respectively, then we must have that $k_{1} \neq k_{2}$ and both are strictly smaller than $j$. This implies that the total number of copies of the length $3^{-j}$ in $\mathcal{L}$ is no greater than $2^{j}$. Thus, the set of lengths of $\mathcal{L}$ is a subset of the lengths of the fractal string generated by a standard middle $1 / 3$ Cantor set of total length 1.

This means that we can rearrange the complementary intervals in the standard middle 1/3 Cantor set so that we obtain the above lengths plus some "extra" lengths. We assume that we do this.

As we mentioned, for any point in the $3^{-n}$ string, the local complex dimensions are of the form

$$
\frac{\ln (2)}{\ln (3)}+\frac{2 \pi i k}{n \ln (3)} \text {. }
$$

Thus, locally we have oscillations with period $2 \pi /(n \ln (3))$ while globally we have oscillations with period $2 \pi / \ln (3)$.

Example 7 Globally Minkowski measurable, (almost) everywhere locally nonmeasurable.

We now given an example of a fractal string which globally has no oscillations (so no poles off of the real axis on the critical line) but everywhere locally it has complex poles, except at one point. For each $n=1,2, \ldots$, let $C_{n}$ be a standard middle $1 / 3$ Cantor set which has been scaled to have total length $n^{-4 / 3}$. We make our string by placing $C_{1}$ starting at the point $0 \in \mathbb{R}$, then leaving a gap of length 1 , then placing $C_{2}$, then leaving a gap of length $2^{-4 / 3}$, then placing $C_{3}$, etc. We must place one more limit point at the far "end" of the set, call this point $\omega$. The total length of this string is $2 \sum_{n} n^{-4 / 3}<8$. The string $C_{n}$ has $2^{k}$ copies of the length $n^{-4 / 3} 3^{-k-1}$, so the zeta function for $C$ is given by

$$
\begin{aligned}
\zeta(s) & =\sum_{n=1}^{\infty} n^{-4 s / 3} 3^{-s} \sum_{k=0}^{\infty} 2^{n} 3^{-n s}+\sum_{n=1}^{\infty} n^{-4 s / 3} \\
& =\sum_{n=1}^{\infty} n^{-4 s / 3} \frac{3^{-s}}{1-2 \cdot 3^{-s}}+\sum_{n=1}^{\infty} n^{-4 s / 3} \\
& =\frac{3^{-s}}{1-2 \cdot 3^{-s}} \sum_{n=1}^{\infty} n^{-4 s / 3}+\sum_{n=1}^{\infty} n^{-4 s / 3}
\end{aligned}
$$




$$
=\zeta_{1}(s) \zeta_{2}(s)+\sum_{n=1}^{\infty} n^{-4 s / 3}=\zeta_{1}(s) \zeta_{2}(s)+\zeta_{2}(s),
$$

where $\zeta_{1}$ is the zeta function for the standard Cantor set and $\zeta_{2}$ is the zeta function for the string with lengths $n^{-4 / 3}$. The critical exponent for $\zeta_{1}$ is $\ln (2) / \ln (3) \approx 0.63$ while the critical exponent for $\zeta_{2}$ is $3 / 4$. Thus, the critical exponent for $\zeta$ is $3 / 4$, where there are no poles with complex dimensions. Thus, globally the string is Minkowski measurable and of dimension 3/4. However, for any $x \in C, x \neq \omega$, and small enough $\epsilon>0$, there is some $n$ so that $B_{\epsilon}(x) \cap C \subset C_{n}$ and thus for any such $\epsilon$ the local zeta function is of the form

$$
\zeta_{\epsilon}(x, s)=\frac{\phi(s)}{1-2 \cdot 3^{-s}},
$$

where $\phi$ is holomorphic. Thus

$$
\mathcal{D}_{\epsilon}(x)=\left\{\frac{\ln (2)}{\ln (3)}+\frac{2 \pi i k}{\ln (3)}: k \in \mathbb{N}\right\} .
$$

Since this is constant for all small $\epsilon$, this is also the set of local complex dimensions at any such point. Thus, locally the string is not Minkowski measurable at any $\omega \neq x \in C$. However, at $\omega$ we see that

$$
\zeta_{\epsilon}(\omega, s)=\frac{3^{-s}}{1-2 \cdot 3^{-s}} \sum_{n \geq N} n^{-4 s / 3}+\sum_{n \geq N} n^{-4 s / 3}
$$

for some $N$. Thus the local complex dimensions at $\omega$ are the same as for the entire string.

The construction in example 7 is interesting to note. If we have two sequences of lengths $a_{n}$ and $b_{n}$ with associated zeta functions $\zeta_{a}$ and $\zeta_{b}$, then the string with lengths $a_{n} b_{m}$ has zeta function $\zeta_{a}(s) \zeta_{b}(s)$, so the zeta function is "multiplicative" in this sense. It is clear that it is "additive" as well.

Constructing a set with the converse property, i.e. globally non-measurable but everywhere locally measurable remains an open question.

Example 8 Nonconvergence of $\mathcal{D}_{\epsilon}(x)$, so a point with no local complex dimensions.

Our final example is one in which there is a point where the local complex dimensions are not defined because of a lack of convergence. Let $r_{k}=1 / 3$ when $k$ is odd and $r_{k}=1 / 4$ when $k$ is even. Furthermore, let $n_{k} \in \mathbb{N}$ grow sufficiently fast so that the solutions $s_{k}>0$ to $r_{k}^{s_{k}}+r_{k}^{s_{k} n_{k}}=1$ are strictly decreasing. Notice that it is always possible to set $n_{2 k+2}=n_{2 k+1}$ but it is necessary to have $n_{2 k+1}>n_{2 k}$. Let $C_{k}$ be a Cantor set with two contractions equal to $r_{k}$ and $r_{k}^{n_{k}}$ and scaled so that $\left|C_{k}\right|=2^{-k}$. Notice that the Hausdorff and box dimension of $C_{k}$ is equal to $s_{k}$. We construct our string $C$ by stacking the $C_{k}$ end-to-end 
from left-to-right and starting from $C_{1}$ and then adding a limit point $\omega$. Then $|C|=1$, the Minkowski dimension of $C$ is $\ln (2) / \ln (3)$ and $C$ is not Minkowski measurable because it has non-real complex poles on the critical line.

We claim that the local complex dimensions do not exist at $\omega$. For all $\epsilon>0$, the critical exponent for $\zeta_{\epsilon}(\omega, s)$ is equal to $s_{k}$ where $k=\min \left\{n: B_{\epsilon}(\omega) \cap C_{n} \neq\right.$ $\emptyset\}$. Thus the complex dimensions of $\zeta_{\epsilon}(\omega, s)$ lie entirely on the line with real part $s_{k}$ and are of the form

$$
s_{k}+\frac{2 \pi i m}{-\ln \left(r_{k}\right)}, \quad m \in \mathbb{N} .
$$

Now we just note that even though $s_{k} \rightarrow 0$, the separation between the complex poles oscilates between $2 \pi / \ln (3)$ and $2 \pi / \ln (4)$, so does not converge.

\section{Acknowledgments}

This work was while FM was visiting JLV at INRIA. FM thanks JLV and INRIA for their hospitality. FM gratefully acknowledges support for this work from the Natural Sciences and Engineering Research Council of Canada (NSERC).

\section{References}

[1] A. Besicovitch and S. Taylor, On the complementary intervals of a linear closed set of zero Lebesgue measure, J. London Math. Soc. 29, 449-459, 1954.

[2] C. Cabrelli, F. Mendivil, U. Molter and R. Shonkwiler, On the Hausdorff h-Measure of Cantor Sets, Pacific Journal of Mathematics, 217(1) $45-59,2004$.

[3] K. Falconer, Fractal Geometry - Mathematical Foundations and Applications, John Wiley, Second Edition, 2003,.

[4] K. Falconer, Techniques in Fractal Geometry, John Wiley, 1997.

[5] B.M. Hambly and M. L. Lapidus, Random Fractal Strings: Their Zeta Functions, Complex Dimensions and Spectral Asymptotics, Trans Amer. Math. Soc. 1(358) 285-314, 2006.

[6] J. Hawkes, Random re-orderings of intervals complementary to a linear set, Q. J. Math, 35, 165-172, 1984.

[7] M. L. Lapidus, Fractal drum, inverse spectral problems for elliptic operators and a partial resolution of the WeylBerry conjecture, Trans. Amer. Math. Soc. 325, 465-529, 1991. 
[8] M. L. Lapidus, Spectral and fractal geometry: From the WeylBerry conjecture for the vibrations of fractal drums to the Riemann zetafunction, in: Differential Equations and Mathematical Physics (C. Bennewitz, ed.), Proc. Fourth UAB Internat. Conf. (Birmingham, March 1990), Academic Press, New York, 151-182, 1992.

[9] M. L. Lapidus, Vibrations of fractal drums, the Riemann hypothesis, waves in fractal media, and the WeylBerry conjecture, in: Ordinary and Partial Differential Equations (B. D. Sleeman and R. J. Jarvis, eds.), vol. IV, Proc. Twelfth Internat. Conf. (Dundee, Scotland, UK, June 1992), Pitman Research Notes in Math. Series, vol. 289, Longman Scientific and Technical, London, 126-209, 1993.

[10] M. L. Lapidus and M. van Frankenhuijsen, Fractal Geometry, Complex Dimensions and Zeta Functions: Geometry and Spectra of Fractal Strings, Springer, New York, 2006.

[11] M. L. Lapidus, J. Lévy Véhel and J. A. Rock, Fractal strings and multifractal zeta functions, Letters in Mathematical Physics (special issue dedicated to Moshe Flato), 88(1-3), 101-129, 2009.

[12] M. L. Lapidus and H. Maier, The Riemann hypothesis and inverse spectral problems for fractal strings, J. London Math. Soc. 52 (2), 1534, 1995.

[13] M. L. Lapidus and E.P.J Pearse, A Tube formula for the Koch snowflake curve with applications to complex dimensions, J. London Math. Soc. 74 (2), 397-414, 2006.

[14] M. L. Lapidus and C. Pomerance, The Riemann zeta-function and the one-dimensional WeylBerry conjecture for fractal drums, Proc. London Math. Soc. 66 (3), 41-69, 1993.

[15] M.L. Lapidus and J. A. Rock, Toward zeta functions and complex dimensions of multifractals, Complex Variables and Elliptic Equations (special issue dedicated to "Fractals"), 54(6), 545-549, 2009.

[16] J. Lévy Véhel and F. Mendivil, Multifractal and higher dimensional zeta functions, Nonlinearity 24(1), 259-276, 2011.

[17] C. Tricot, Curves and Fractal Dimension, Springer Verlag, New York, 1995.

[18] C. Tricot, Douze définitions de la densité logarithmique, C. R. Acad. Sci. Paris Sér. 1 Math. 293, 549-552, 1981. 